\title{
Cosmic Initial Singularities in a Single Repeating Universe as Opposed to Their Behavior in a Multiverse
}

\author{
Andrew Beckwith \\ Department of Physics, Chongqing University, Chongqing, China \\ Email: abeckwith@uh.edu
}

How to cite this paper: Beckwith, A. (2017) Cosmic Initial Singularities in a Single Repeating Universe as Opposed to Their Behavior in a Multiverse. Journal of High Energy Physics, Gravitation and Cosmology, 3, 467-492.

https://doi.org/10.4236/jhepgc.2017.33037

Received: April 24, 2017

Accepted: July 28, 2017

Published: July 31, 2017

Copyright $\odot 2017$ by author and Scientific Research Publishing Inc. This work is licensed under the Creative Commons Attribution International License (CC BY 4.0).

http://creativecommons.org/licenses/by/4.0/

\begin{abstract}
When the initial radius of the universe is set in four dimensions and if there is only ONE repeating universe, then the initial radii of the universe is $R \rightarrow 0$ or gets very close to zero if we use the Einstein Equations modified by Stoica. The Einstein Equations are reset by Stoical in a formalism which removes in four dimensions, the big bang singularity pathology. So then the reason for Planck length no longer holds. This manuscript assumes a repeating single universe. We present entanglement entropy in the early universe with a shrinking scale factor, due to Muller and Lousto, and show that there are consequences due to initial entangled $S_{\text {Entropy }}=0.3 r_{H}^{2} / a^{2}$ for a time dependent horizon radius $r_{H}$ in cosmology, with (flat space conditions) $r_{H}=\eta$ for conformal time. Even if the 3-dimensional spatial length goes to zero. Our new manuscript presentation sets as a starting point a cosmology with a non-zero $\Lambda$ vacuum energy. The non-zero $\Lambda$ vacuum energy, initial configuration of the universe permits us to keep in an information theory stand point (information theory), computational bits for our configuration of cosmological expansion. This assemblage of computational bits occurs in cosmological evolution even if in an initial four-dimensional cosmology, we have the initial radii of the universe $R \rightarrow 0$. We also find that in the case of a multiverse, such considerations will not hold and that cosmic singularities have a more different characteristic in the multiverse setting than in the single universe repeated over and over again, i.e. using an argument borrowed and modified from Kauffman, the multiverse will not mandate "perfect" singularities. The existence of a multiverse may allow for non zero singularities in lieu with the Kauffman argument cited at the end of the document, plus the lower pre big bang temperatures which may allow for the survival of gravitons just before the onset of the cosmological expansion phase, if a multiverse exists embedding our present universe.
\end{abstract}




\section{Keywords}

Fjortoft Theorem, Thermodynamic Potential, Matter Creation, Vacuum Energy Non Pathological Singularity Affecting Einstein

Equations, Planck Length, Braneworlds

\section{Introduction}

We first examine what is to be expected in the four-dimensional case as to what happens if there is a single repeating universe. In such a situation, one can employ the following argument as to a singularity with the aforementioned behavior as given below. First before doing it, we investigate via simple arguments involving scaling arguments for the Friedman equations what to expect in the case that the cosmological "constant" is indeed a constant or has temperature dependence as $\mathrm{T}$ to the beta power, according to formalism developed by Park et al. [1]. In doing so, a case can be made using the Weinberg argument [2] that if there is a high initial background "viscosity" (for graviton propagation), there is a high initial temperature. This high temperature would be consistent with the modus operandi of a single repeating universe, done again and again. To make the point of this, we also can refer to the Penrose CCC [3] hypothesis as yet another way to delineate this same repeating universe, with black holes in four dimensions, i.e. in the case of the single repeating universe, one may use the Stoica convention [4] as to non pathological singularities, or a near singularity at the beginning of space-time.

The situation changes if we have a multiverse. Here, the behavior of viscosity changes fundamentally in terms of its contribution to temperature, and this in turn has manifest implications as to possible avoidance of singularities in initial space time. To set the frame work for doing this, we will generalize the Penrose CCC hypothesis [3] in a way presented by the author in San Marino, Italy, and other places. First now, let us look at the single repeating universe case and comment upon it.

The final part will be a summary in Section 13 below which states a generalized treatment as to how the singularity is avoided in the multiverse with reccomendations as to future research in Section 14.

\section{If There Is a Single Repeating Universe, What Can We Expect in Terms of Entropy and Singularies? Case Written Below}

This first part of the article is to investigate what happens physically if there is a non pathological singularity in terms of Einstein's equations at the start of space-time if there is a single repeating universe. This eliminates the necessity of having then put in the Planck length since then there would be no reason to have a minimum non-zero length. The reasons for such a proposal come from [4] by Stoica who may have removed the reason for the development of Planck's length 
as a minimum safety net to remove what appears to be unavoidable pathologies at the start of applying the Einstein equations at a space-time singularity, and are commented upon in this article. $\rho \sim H^{2} / G \Leftrightarrow H \approx a^{-1}$ in particular is remarked upon. This is a counterpart to Fjortoft theorem as cited in Appendix I. The idea is that entanglement entropy will help generate bits, due to the presence of a vacuum energy, as derived at the end of the article, and the presence of a vacuum energy non-zero value, is necessary for cosmological evolution. Before we get to that creation of what is a necessary creation of vacuum energy conditions we refer to constructions leading to extremely pathological problems which [4] could lead to minus the presence of initial non zero vacuum energy, due to the inputs of reference [5] which also adds more elaboration on this idea of necessary additions to the initial conditions of the repeating universe.

Note a change in entropy formula given by Lee [6] about the inter relationship between energy, entropy and temperature as given by

$$
m \cdot c^{2}=\Delta E=T_{U} \cdot \Delta S=\frac{\hbar \cdot a}{2 \pi \cdot c \cdot k_{B}} \cdot \Delta S
$$

Lee's formula is crucial for what we will bring up in the latter part of this document. Namely that changes in initial energy could effectively vanish if [4] is right, i.e. Stoica removing the non pathological nature of a big bang singularity. That is, unless entanglement entropy is used.

If the mass $m$, i.e. for gravitons is set by acceleration (of the net universe) and a change in enthropy $\Delta S \sim 10^{38}$ between the electroweak regime and the final entropy value of, if $a \cong \frac{c^{2}}{\Delta x}$ for acceleration is used, so then we obtain

$$
S_{\text {Today }} \sim 10^{88}
$$

Then we are really forced to look at Equation (1) as a paring between gravitons (today) and gravitinos (electro weak) in the sense of preservation of information.

Having said this note by extention $\rho \sim H^{2} / G \Leftrightarrow H \approx a^{-1}$. As $\rho$ changes due to $\rho \sim H^{2} / G$ and $R_{\text {initial }} \sim \frac{1}{\#} \ell_{N g}<l_{\text {Planck }}, t$ hen $a$ is also altered i.e. goes to zero.

What will determine the answer to this question is if $\Delta E_{\text {initial }}$ goes to zero if $R_{\text {initial }} \rightarrow 0$ which happens if there is no minimum distance mandated to avoid the pathology of singularity behavior at the heart of the Einstein equations. In doing this, we avoid using the energy $E \rightarrow 0^{+}$situation, i.e. of vanishing initial space-time energy, and instead refer to a nonzero energy, with $\Delta E_{\text {initial }}$ instead vanishing. In particular, the Entanglement entropy concept as presented by Muller and Lousto [7] is presented toward the end of this manuscript as a partial resolution of some of the pathologies brought up in this article before the entanglement entropy section. No matter how small the length gets, $S_{\text {entropy }}$ if it is entanglement entropy, will not go to zero. The requirement is that the smallest length of time, $t$, rescaled, does not go to zero. This preserves a minimum non zero $\Lambda$ vacuum energy, and in doing so keep non zero amounts of initial bits, 
for computational bits cosmological evolution even if $R_{\text {initial }} \rightarrow 0$.

Before doing that, we review $\mathrm{Ng}[8]$ and his quantum foam hypothesis to give conceptual underpinnings as to why we later even review the implications of entanglement. Entropy, i.e. the concept of bits and computations is brought up because of applying energy uncertainty, as given by [8] and the Margolis theorem appears to indicate that the universe could not possibly evolve if [8] is applied, in a 4 dimensional closed universe. This bottle neck as indicated by Ng's [8] formalism is even more striking in the author's end of article proof of the necessity of using entanglement entropy in lieu of the conclusion involving entanglement entropy, which can be non zero, even if $R_{\text {initial }} \rightarrow 0$ provided therre is a minimum non zero time length.

\section{Review of $\mathrm{Ng}$, [8] with Comments}

First of all, $\mathrm{Ng}$ [8] refers to the Margolus-Levitin theorem with the rate of operations $<E / \hbar \Rightarrow \#$ operations $<E / \hbar \times$ time $=\frac{M c^{2}}{\hbar} \cdot \frac{l}{c}$. Ng wishes to avoid blackhole formation $\Rightarrow M \leq \frac{l c^{2}}{G}$. This last step is not important to our view point, but we refer to it to keep fidelity to what $\mathrm{Ng}$ brought up in his presentation. Later on, $\mathrm{Ng}$ refers to the \# operations $\leq\left(R_{H} / l_{P}\right)^{2} \sim 10^{123}$ with $R_{H}$ the Hubble radius. Next Ng refers to the \#bits $\propto[\text { \# operations] }]^{3 / 4}$. Each bit energy is $1 / R_{H}$ with $R_{H} \sim l_{P} \cdot 10^{123 / 2}$.

The key point as seen by $\mathrm{Ng}[8]$ and the author is in

$$
\text { \# bits } \sim\left[\frac{E}{\hbar} \cdot \frac{l}{c}\right]^{3 / 4} \approx\left[\frac{M c^{2}}{\hbar} \cdot \frac{l}{c}\right]^{3 / 4}
$$

Assuming that the initial energy $E$ of the universe is not set equal to zero, which the author views as impossible, the above equation says that the number of available bits goes down dramatically if one sets $R_{\text {initial }} \sim \frac{1}{\#} \ell_{N g}<l_{\text {Planck }}$ ? Also $\mathrm{Ng}$ writes entropy $S$ as proportional to a particle count via $N$.

$$
S \sim N \cong\left[R_{H} / l_{P}\right]^{2}
$$

We rescale $R_{H}$ to be

$$
\left.R_{H}\right|_{\text {rescale }} \sim \frac{l_{N g}}{\#} \cdot 10^{123 / 2}
$$

The upshot is that the entropy, in terms of the number of available particles drops dramatically if \# becomes larger.

So, as $R_{\text {initial }} \sim \frac{1}{\#} \ell_{N g}<l_{\text {Planck }}$ grows smaller, as \# becomes larger:

1) The initial entropy drops;

2) The number of bits initially available also drops.

The limiting case of Equation (4) and Equation (5) in a closed universe, with no higher dimensional embedding is that both would almost vanish, i.e. appear to go to zero if \# becomes very much larger. The question we have to ask is 
would the number of bits in computational evolution actually vanish?

\section{Does It Make Sense to Talk of Vacuum Energy If $\boldsymbol{R}_{\text {initial }} \neq 0$ Is Changed to $\boldsymbol{R}_{\text {initial }} \rightarrow \mathbf{0}$ ? Only Answerable Straightforwardly If an Embedding Superstructure Is Assigned. Otherwise Difficult}

Unless one is using entanglement entropy which is non zero even if $R_{\text {initial }} \rightarrow 0$, And this uses the ideas of [8], although in a non obvious fashion. And it is Otherwise difficult. Unless one is using entanglement entropy which is non zero even if $R_{\text {initial }} \rightarrow 0$. Note that we summarize what may be the high lights of this inquiry leading to the present paper as follows.

1) One could have the situation if $R_{\text {initial }} \rightarrow 0$ of an infinite point mass, if there is an initial nonzero energy in the case of four dimensions and no higher dimensional embedding even if [4] goes through verbatim. The author sees this as unlikely. The infinite point mass construction is verbatim if one assumes a closed universe, with no embedding superstructure and no entanglement entropy. Note this appears to nullify the parallel brane world construction used by Durrer [9]. The author, in lieu of the manuscript sees no reason as to what would perturb this infinite point structure, so as to be able to enter in a big bang era. In such a situation, one would not have vacuum energy unless entanglement entropy was used. That is unless one has a non zero entanglement entropy As given by Volovik [10] present even if $R_{\text {initial }} \rightarrow 0$. See [11] for a smilar argument.

2) The most problematic scenario. $R_{\text {initial }} \rightarrow 0$ and no initial cosmological energy. i.e. this in a 4 dimensional closed universe. Then there would be no vacuum energy at all. initially. A literal completely empty initial state, which is not held to be viable by Volovik [10].

3) If additional dimensions are involved in beginning cosmology, than just 4 dimensions will lead to physics which may give credence to other senarios. One scenario being the authors speculation as to initial degrees of freedom reaching up to 1000 , and the nature of a phase transition from essentially very low degrees of freedom, to over 1000 as speculated by the author in 2010 [12].

4) What the author would be particularly interested in knowing would be if actual semiclassical reasoning could be used to get to an initial prequantum cosmological state. This would be akin to using [13], but even more to the point, using [14] and [15], with both these last references relevant to forming Planck's constant from electromagnetic wave equations. The author points to the enormous Electromagnetic fields in the electroweak era as perhaps being part of the background necessary for such a semiclassical derivation, plus a possible Octonionic space-time regime, as before inflation flattens space-time, as forming a boundary condition for such constructions to occur [16]. This is still using the ideas of [8] which we will amplify upon, later:

The relevant template for examining such questions is given in following $\mathrm{Ta}$ ble 1 as printed below. 
Table 1. Time interval dynamical consequences do QM/WdW apply.

\begin{tabular}{|c|c|c|}
\hline Just before Electroweak era & $\begin{array}{l}\text { Form } \hbar \text { from early E \& M } \\
\text { fields, and use Maxwell's } \\
\text { Equations with necessary to } \\
\text { implement boundary } \\
\text { conditions created from } \\
\text { change from Octonionic } \\
\text { geometry to flat space }\end{array}$ & NO \\
\hline Electro-Weak Era & $\begin{array}{l}\hbar \text { kept constant due to } \\
\text { Machian relations }\end{array}$ & YES \\
\hline $\begin{array}{l}\text { Post Electro-Weak Era to } \\
\text { today }\end{array}$ & $\begin{array}{l}\hbar \text { kept constant due to } \\
\text { Machian relations }\end{array}$ & $\begin{array}{c}\text { YES } \\
\text { Wave function of Universe }\end{array}$ \\
\hline
\end{tabular}

5) The meaning of Octonionic geometry prior to the introduction of quantum physics presupposes a form of embedding geometry and in many ways is similar to Penrose's cyclic conformal cosmology speculation.

6) It is striking how a semiclassical argument can be used to construct Table 1 below. In particular, we look at how Planck's constant is derived, as in the electroweak regime of space-time, for a total derivative [14] [15]

$$
E_{y}=\frac{\partial A_{y}}{\partial t}=\omega \cdot A_{y}^{\prime}(\omega \cdot(t-x))
$$

Similarly [10] [11]

$$
B_{z}=-\frac{\partial A_{y}}{\partial x}=\omega \cdot A_{y}^{\prime}(\omega \cdot(t-x))
$$

The A field so given would be part of the Maxwell's equations given by [9] as, when [ ] represents a D'Albertain operator, that in a vacuum, one would have for an A field [14] [15]

$$
[] A=0
$$

And for a scalar field $\phi$

$$
[] \phi=0
$$

Following this line of thought we then would have an energy density given by, if $\varepsilon_{0}$ is the early universe permeability [14]

$$
\eta=\frac{\varepsilon_{0}}{2} \cdot\left(E_{y}^{2}+B_{z}^{2}\right)=\omega^{2} \cdot \varepsilon_{0} \cdot A_{y}^{\prime 2}(\omega \cdot(t-x))
$$

We integrate Equation (10) over a specified $\mathrm{E}$ and $\mathrm{M}$ boundary, so that, then we can write the following condition namely [14] [15]

$$
\iiint \eta \mathrm{d}(t-x) \mathrm{d} y \mathrm{~d} z=\omega \varepsilon_{o} \iiint A_{y}^{\prime 2}(\omega \cdot(t-x)) \mathrm{d}(t-x) \mathrm{d} y \mathrm{~d} z
$$

Equation (11) would be integrated over the boundary regime from the transition from the Octonionic regime of space time, to the non Octonionic regime, assuming an abrupt transition occurs, and we can write, the volume integral as representing [14] [15]

$$
E_{\text {gravitational-energy }}=\hbar \cdot \omega
$$


Then by applying [14] [15] we get $\hbar$ formed by semiclassical reasons In semi classical reasoning similar to [13]

$$
\hbar(t) \underset{\text { Apply-Machs-Relations }}{\longrightarrow} \hbar \text { (Constant value) }
$$

The question we can ask, is that can we have a prequantum regime commencing for Equation (11) and Equation (12) for $\hbar$ if $R_{\text {initial }} \sim \frac{1}{\#} \ell_{N g}<l_{\text {Planck }}$ ? And a closed 4 dimensional universe? If so, then what is the necessary geometrial regime of space-time so that the integration performed in (11) can commence properly? Also, what can we say about the formation of Equation (12) above, as a number, \# gets larger and larger, effectively leading to. Also, with an Octonionic geometry regime which is a pre quantum state [16].

In so many words, the formation period for $\hbar$ is our pre-quantum regime. Table 1 could even hold if $R_{\text {initial }} \rightarrow 0$ but that the 4 dimensional space-time exhibiting such behavior is embedded in a higher dimensional template. That due to $R_{\text {initial }} \rightarrow 0$ not removing entanglement entropy as is discussed near the end of this article.

\section{If $R_{\text {initial }} \rightarrow 0$ Then If There Is an Isolated, Closed Universe, There Is a Disaster Unless One Uses Entanglement Entropy}

One does not have initial entropy, and the number of bits initially disappears. That is if one is not using entanglement entropy, as will be examined at the end of this article. Abandoning the idea of a completely empty universe, this unperturbed point of matter-energy appears to be a recipede for a static point with no perturbation, as may be the end result of applying Fjortoft theorem [17] to the thermodynamic potential as given in [18], i.e. the non definitive anwer for fufillment of criteria of instability by applying Fjortoft's theorem [17] to the potential [18] leading to no instability as given by the potential given in [18] may lead to a point of space-time with no change, i.e. a singular point with "infinite" mass which does not change at all. This issue will be reviewed in [19] by use of a different procedure, i.e. a so called nonsingular universe construction. To get there we will first of all review an issue leading up to implimentation of [19]. These are non-Singular cosmologies of the sort presented in [19] by Gao.

\section{Can an Alternative to a Minimum Length Be Put in? Consider the Example of Planck Time as the Minimal Component, Not Planck Length}

From J. Dickau, [20] the following was given to the author, as a counter point to $R_{\text {initial }} \rightarrow 0$ leading to a disaster.

"If we examine the Mandelbrot Set along the Real axis, it informs us about behaviors that also pertain in the Quaternion and Octonic case-because the real axis is invariant over the number types. If numbers larger than 0.25 are squared and summed recursively (i.e. $-Z=Z^{2}+c$ ) the result will blow up, but numbers 
below this threshold never get to infinity, no matter how many times they are iterated. But once space-like dimensions are added-i.e. an imaginary compoentthe equation blows up exponentially, faser than when iterated".

Dickau concludes:

"Anyhow there may be a minimum (space-time length) involved but it is probably in the time direction and the equation proceeds to blow up exponentially, faster than when it is iterated in space-time". This is a crucial datum which Dickau viewed as an overlooked dynamic in this evolutionary process.

This is a counter pose to the idea of minimum length, looking at a beginning situation with a crucial parameter $R_{\text {initial }}$ even if the initial time step is "put in by hand". First of all, look at [7], if $E$ is $M$, due to setting $c=1$, then

$$
\Delta E_{\text {initial }} \approx 4 \pi \rho\left(R_{\text {initial }}\right)^{2} \Delta R_{\text {initial }}
$$

Everything depends upon the parameter $R_{\text {initial }}$ which can go to zero. We have to look at what Equation (14) tells us, even if we have an initial time step for which time is initially indeterminate, as given by a redoing of Mitra's $g_{00}$ formula Equation [11] which we put in to establish the indeterminacy of the initial time step if quantum processes hold.

$$
\left(g_{00}=\exp \left[\frac{-2}{1+(\rho(t) / p(t))}\right]\right) \underset{\rho+p=0}{\longrightarrow} 0
$$

What Dickau [20] is promoting is, that the Mandelbrot set, if applicable to early universe geometry, that what the author wrote, with $R_{\text {initial }} \sim \frac{1}{\#} \ell_{N g}<l_{\text {Planck }} \underset{\# \neq \infty}{\longrightarrow}$ small-value potentially going to zero, is less important than a minimum time length. The instability issue is reviewed in Appendix II for those who are interested in the author's views as to lack proof of instability. It uses [18] which the author views as THE reference as far as thermodynamic potentials and the early universe.

\section{Muller and Lousto Early Universe Entanglement \\ Entropy, and Its Implications. Solving the Spatial \\ Length Issue, Provided a Minimum Time Step Is \\ Preserved in the Cosmos, in Line with Dickau's Suggestion}

We look at [7] $S_{\text {Entropy }}=0.3 r_{H}^{2} / a^{2}$ for a time dependent horizon radius $r_{H}$ in cosmology.

Equation (16) above was shown by the author to be fully equivalent to

$$
S_{\text {Entropy }}=0.3 r_{H}^{2} / a^{2} \sim \frac{0.3}{a^{2}} \exp \left[-t \cdot \sqrt{\frac{\Lambda}{3}}\right]
$$

i.e.

$$
\left[-t \sqrt{\frac{\Lambda}{3}}\right] \sim \ln \left(\frac{a^{2}}{0.3} \cdot S_{\text {entropy }}\right)
$$

So, then one has 


$$
\Lambda \approx \frac{3}{t^{2}} \cdot\left[\ln \left(\frac{a^{2}}{0.3} \cdot S_{\text {entropy }}\right)\right]^{2}
$$

No matter how small the length gets, $S_{\text {entropy }}$ if it is entanglement entropy, will not go to zero. The requirement is that the smallest length of time, $t$, re scaled does not go to zero. This preserves a minimum non zero $\Lambda$ vacuum energy, and in doing so keep the non zero initial bits, for computational bits contributions to evolving space time behavior even if $R_{\text {initial }} \rightarrow 0$.

\section{Reviewing a Suggestion as to How to Quantify the Shrinkage of the Scale Factor and Its Connections with Entanglement Entropy}

We are given by [19], by Gao a procedure to follow if there is a non singular universe, which is a template as to how to evaluate scale factor $a$ against time scaled over Planck time, with the following important results.

$$
\ln _{e} a+\frac{a^{6}}{6}+\frac{2 \cdot a^{3}}{3}=\sqrt{\frac{8 \pi}{3}} \cdot \frac{t}{t_{\text {Planck }}}
$$

Two time and scale factor values in tandem particularly stand out. Namely,

$$
a \sim \frac{a_{\text {scale }}}{\left[a_{\text {Planck }} \sim 10^{-25}\right]} \equiv 1.344 \Leftrightarrow t \propto t_{\text {Planck }} \sim 5.4 \times 10^{-44} \mathrm{sec}
$$

Also

$$
a \sim \frac{a_{\text {scale }}}{\left[a_{\text {Planck }} \sim 10^{-25}\right]} \equiv 0.7414 \Leftrightarrow t \propto 0^{+}
$$

The main thing we can take from this, is to look at the inter-relationship of how to pin down an actual initial Hubble "constant" expansion parameter, where we look at:

$$
1.813=\exp \left(H_{\text {Planck }} \cdot t_{\text {Planck }}\right) \Leftrightarrow H_{\text {Planck }}=\frac{\ln _{e}(1.813)}{t_{\text {Planck }}}
$$

We set $\Lambda \approx \frac{3}{t^{2}} \cdot\left[\ln e\left(\frac{a^{2}}{0.3} \cdot S_{\text {entropy }}\right)\right]^{2}$, which is predicated upon, if the time is close to Planck time the initial maximal density of

$$
\rho_{\text {Planck }} \sim 5.2 \times 10^{96} \mathrm{~kg} / \mathrm{m}^{3}
$$

And length given by

$$
\text { Length }(\text { Planck })=l_{\text {Planck }} \sim 1.6 \times 10^{-35} \text { meters }
$$

So (24) is implying that the amount of matter in a region of space $\left(l_{\text {Planck }}\right)^{3}$ is initially about

$$
\rho_{\text {initial }} \sim 2 \times 10^{-10} \mathrm{~kg} \sim 2 \times 10^{-7} \text { grams }
$$

Using $1 \mathrm{GeV} / \mathrm{c}^{2}=1.783 \times 10^{-27} \mathrm{~kg}$ means that (26) above is

$$
\rho_{\text {initial }} \sim 2 \times 10^{-10} \mathrm{~kg} \sim 2 \times 10^{-7} \text { grams } \sim 10^{+17} \mathrm{GeV}
$$


Then if

$$
10^{+17} \mathrm{GeV} \sim \Lambda \approx \frac{3}{t^{2}} \cdot\left[\ln _{e}\left(\frac{a^{2}}{0.3} \cdot S_{\text {entropy }}\right)\right]^{2}
$$

It will lead to

$$
10^{+17} \mathrm{GeV} \times 10^{-70} \sec ^{2} \sim\left[\ln \left(\frac{a^{2}}{0.3} \cdot S_{\text {entropy }}\right)\right]
$$

Then, to first order, one is looking at Initial entropy to get a non zero but definite vacuum energy as leading to an entanglement entropy of about (just before the electro weak regime)

$$
S_{\text {entropy }} \sim 1 / a^{2} \sim 10^{20}-10^{40}
$$

\section{Reviewing the Geometry for Embedding Equation (29) Above}

In line with Stoica [4] and [9] and [21], In addition to this, the construction should make use of [22], i.e. the quantum measure as designated by Surya. Note that the idea behind this has a counterpart as given by the author in [23]. Note that [24] and [25] denote the brane theory version of cosmology as well as Cosmology using Brane theory as a starting point.

We are then shrinking the minimum length and referring to both Equation (29) and Equation (27), and the idea is to use a surface area treatment as to getting the initial entropy values as given in Equation (29). To do so, the author looks at the following diagram:

Brane world dynamics in the case of a single, repeating universe, as opposed to a multiverse.

Now for a review of Figure 1. The two branes given at $y_{b}$ and $y_{s}$ refer to the two Brane world states, especially in line with [24] [25]. The first one, namely $y_{b}$ is the brane where our physical universe lives in, and is embedded in. If one uses this construction, with higher dimensions than just 4 dimensions, then it is possible to have a single point in 4 dimensional space as a starting point to a tangential sheet which is part of an embedding in more than 4 dimensions. Along the lines of having a 4 dimensional cusp with its valley (lowest) point in a more than 4 dimensional tangential surface. The second brane is about $10^{-30}$ centimeters away from the brane our physical world lives in, and moves closer

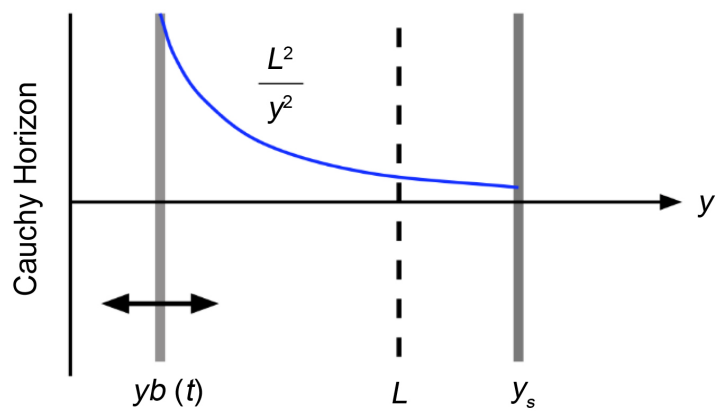

Figure 1. From [9]. 
to our own brane in the future, leading to a slapping of the two branes together about a trillion years ahead in our future [24] [25]. The geometry we are referring to with regards to embedding is in the first brane $y_{b}$. [9] uses this geometry to have graviton production which the author has used to model Dark Energy [21].

\section{0. $1^{\text {st }}$ Conclusion When Looking at ONE Repeating Universe. Making Computational Bits, via (19)}

As stated by $\mathrm{Ng}$ [8], the idea would be to have to give imputs into Equation (3) i.e.

$$
\text { \# bits } \sim\left[\frac{E}{\hbar} \cdot \frac{l}{c}\right]^{3 / 4} \approx\left[\frac{M c^{2}}{\hbar} \cdot \frac{l}{c}\right]^{3 / 4}
$$

Here in this case, even if the spatial contribution, due to [4] goes to zero, the idea would be to have the time length non zero so as to have a space-time version of $l$ non zero. This would also be in tandem with calling $E$, in (3) as proportional to $\Lambda \approx \frac{3}{t^{2}} \cdot\left[\ln \left(\frac{a^{2}}{0.3} \cdot S_{\text {entropy }}\right)\right]^{2}$, where if the time is Planck time, in minimum value, and $S_{\text {entropy }} \sim 1 / a^{2} \sim 10^{20}-10^{40}$ in value, one would have before the electro-weak an input into $E$, which would require an entropy (entanglement).

What remains to be seen is, if there is a geometric sheet in more than 4 dimensions, allowing for non zero time, as argued for $\Lambda \approx \frac{3}{t^{2}} \cdot\left[\ln \left(\frac{a^{2}}{0.3} \cdot S_{\text {entropy }}\right)\right]^{2}$, even if the spatial componet goes to zero, according to [4]. We suggest an update as to what was written by Lloyd [26]

$$
I=S_{\text {total }} / k_{B} \ln 2=[\# \text { operations }]^{3 / 4}=\left[\rho \cdot c^{5} \cdot t^{4} / \hbar\right]^{3 / 4}
$$

when [27]

$$
\rho \equiv T^{00} \sim \Lambda_{\text {vacuum-energy }}
$$

While doing this, a good thing to do, would be to keep in mind the four dimensional version of vacuum energy as given by Park, [1] namely

$$
\Lambda_{4-\operatorname{dim}} \approx c_{2} \cdot T^{\beta}
$$

As well as the transition given by a combination of [1], with [28], Barvinskey et al.

$$
\Lambda_{4-\text { dim }} \propto c_{2} \cdot T \underset{\text { graviton-production }}{\longrightarrow} 360 \cdot m_{P}^{2} \ll c_{2} \cdot\left[T \approx 10^{32} \mathrm{~K}\right]
$$

Quantifying the above, and giving it experimental proof, via detector technology may allow us to investigate an old suggestion by the author as to four dimension and five dimensional vacuum energy which was given for small time values $t \approx \delta^{1} \cdot t_{P}, \quad 0<\delta^{1} \leq 1$ and for temperatures sharply lower than $T \approx 10^{12}$ Kelvin , Beckwith [22], where for a positive integer $n$ 


$$
\frac{\Lambda_{4-\operatorname{dim}}}{\left|\Lambda_{5-\operatorname{dim}}\right|}-1 \approx \frac{1}{n}
$$

In particular, the author is interested in investigating if the following is true, i.e. look at an argument provided by Padmanabhan [29] and [30], leading to the observed cosmological constant value suggested by Park [1]. Assume that $l_{P} \sim 10^{-33} \mathrm{~cm} \underset{\text { Quantum-Gravity-threshold }}{\longrightarrow} \tilde{N}^{\alpha} \cdot l_{P}$, but that when we make this substitution that $1 \leq \tilde{N}^{\alpha} \leq 10^{2} \quad[30]$

$$
\rho_{V A C} \sim \frac{\Lambda_{\text {observed }}}{8 \pi G} \sim \sqrt{\rho_{U V} \cdot \rho_{I R}} \sim \sqrt{l_{\text {Planck }}^{-4} \cdot l_{H}^{-4}} \sim l_{\text {Planck }}^{-2} \cdot H_{\text {observed }}^{2}
$$

i.e. looking at if

$$
\Delta \rho \approx \text { a dark energy density } \sim H_{\text {observed }}^{2} / G
$$

Now to make it more interesting:

We can replace $\Lambda_{\text {observed }}, H_{\text {observed }}^{2}$ by $\Lambda_{\text {initial }}, H_{\text {initial }}^{2}$. In addition we may look at inputs from the initial value of the Hubble parameter to get the necessary e folding needed for inflation, according to

$$
\begin{aligned}
& E \text {-foldings }=H_{\text {initial }} \cdot\left(t_{\text {End of inf }}-t_{\text {beginning of inf }}\right) \equiv N \geq 100 \\
& \Rightarrow H_{\text {initial }} \geq 10^{39}-10^{43}
\end{aligned}
$$

Leading to

$$
a(\text { End-of-inf }) / a(\text { Beginning-of-inf }) \equiv \exp (N)
$$

If we set $\Lambda_{\text {initial }} \sim C_{1} \cdot\left[T \sim 10^{32}\right.$ Kelvin $]$ implying a very large initial cosmological constant value, we get in line with what Park suggested for times much less than the Planck interval of time at the instant of nucleation of a vacuum state

$$
\Lambda_{\text {initial }} \sim\left[10^{156}\right] \cdot 8 \pi G \approx \text { huge number }
$$

Note that the following ideas, as given in Crowell, [31] as to brane theory construction are assumed as possible entries into this set up for the value of Equation [32], and that in addition, [33] as to a cyclic repeating universe are also assumed to be contenders into our evolving space-time model. So then we will reference, afterwards what we think is an important issue about Equation (39).

Question. Do we always have this value of Equation (39)? At the onset of Inflation? When we are not that far away from a volume of space characterized by $l_{P}^{3}$, or at most 100 or so times larger? Contemporary big bang theories imply this. i.e. a very high level of thermal energy. We need to ask if this is something which could be transferred from a prior universe, i.e. could there be a pop up nucleation effect, i.e. emergent space time? This question is what should be investigated thoroughly. Appendix III and Appendix IV give suggestions which the author has thought of which may contribute to, if anything, models of how instantons from a prior universe may be transmitted to our present universe, i.e. Appendix $\mathrm{V}$ which is based in part on what Wesson formulated as to five dimensional universe constructions, and instantons [34]. The very interesting top- 
ic of vacuum fluctuations in such space-time has also been reviewed briefly in Appendix VI, and Appendix VII.

\section{What If We Have a Multiverse? Argue Then that the Above Methodology Should Be Modified. i.e. Consider the Following Scaling of Quantities from the Friedman Equation}

Using the formalism given in Peacock [35], page 80 we can make the following scaling argument which will prove useful as to the divergence of the multiverse case, and how there is no longer a convenient singularity to refer to, via Stoica [4] or anyone else.

Peacock, has it that one can write as follows, namely for a scale factor, with $a_{0}$.

infinitesimally small and representing the intial scale factor in pre Planckian space time

$$
a(t)=a_{0} \exp [\tilde{\tilde{H}} \cdot t]
$$

Here, the main evolving factor to consider is, Peacock [35], page 80 with $\Lambda_{\text {cos-const }}$ from Equation (32) above. If so then we have to look at one datum which will be important. Mainly, how the temperature changes

$$
\tilde{\tilde{H}}=\sqrt{\frac{\Lambda_{\text {cos-const }} \cdot c^{2}}{3}}
$$

If the term beta in the coefficient of temperature, $T$, is zero, we have merely the Einstein vanilla Cosmological constant. If the term beta in the coefficient of temperature, $T$, is not zero, then we should take a good look at what is done in Weinberg [2] where there is a concerted effort to mix in background viscosity = But first take note of the Grischuck [36] expression as to GW frequency in the hign end, with Temperature approaching Planck Tempure values, with behavior due to space-time temperature dependence as given by: $M=$ mass of "universe" initially, and $R=$ radius of initial dimensions, with Planck temperature values giving for a single universe, and four dimensions

$$
f_{\text {Peak }} \approx\left(10^{-3} \mathrm{~Hz}\right) \cdot\left[\frac{T}{T \mathrm{eV}}\right] \sim 10^{10} \mathrm{~Hz} \approx \sqrt{\frac{M}{M_{\text {solar-mass }}}} \cdot \sqrt{\frac{90 \mathrm{~km}}{R}}
$$

Then in doing this, we will also consider the case where the temperature is low, which we claim is due to a multiverse, and this will be leading to, for ultra low initial temperatures, for the pre big bang, namely if there is a multiverse having

$$
f_{\text {Peak }} \approx\left(10^{-3} \mathrm{~Hz}\right) \cdot\left[\frac{T}{T \mathrm{eV}}\right] \sim 10^{-18}-10^{-10} \mathrm{~Hz}
$$

Furthermore, we can write from Page 163 of Penrose [3], that, if $N \sim S$ (entropy) as given by $\mathrm{Ng}$ [8] for a regular single repeating universe structure, that we will have, for a cyclical universe the following power law relationship. Note that in Equation (44) the abbreviation of F. T. stands for field theory. To do this we will 
utilize the background in model construction as see by [36] [37] [38] [39], and [32] which the author views as essential reading a cosmologist needs to attend to in order to avoid the annoyance of the anthropic principle, which is semi religious and which should be avoided, and then go to our important next result, Equation (44) below

$$
\left.\Lambda\right|_{\text {Today }} \text { [vacuum-energy] }\left.\sim \frac{c^{3}}{N^{6} G \hbar} \equiv N^{-6}\right|_{\text {value-Today }} \approx 10^{-120} \sim 1 / S_{\text {initially }}^{6} \quad \text { times }\{\mathrm{FT}
$$

calculated version of Vacuum energy\}

i.e. that the cosmological constant is set by the initial release of, maybe, gravitons. i.e. and also note that if one does this that one is able to state that initially low entropy will lead to a tendency, later to a scaled cosmological constant which is todays value.

The question in reaching Equation (44) is, if entropy is commensurate with graviton production as brought up by both Beckwith [21] and Giovannini [37] that then one is looking at thermally induced GW production which may indeed impact the cosmological constant. The question to ask is the following reasonable? i.e. as given by Giovannini [37]

$$
\begin{aligned}
S_{\text {Gravtion-Today'.era }} & \propto V \int_{10^{-19} \mathrm{Hertz}}^{10^{11} \mathrm{Hertz}} r(v) \cdot v^{2} \mathrm{~d} v \\
& \cong\left(10^{30}\right)^{3} \cdot\left(\frac{H_{1}}{M_{\text {Planck-mass }}}\right) \propto 10^{90} \\
& \sim\left(\text { difference of } 10^{11} \text { to } 10^{-19} \text { Hertz }\right)^{3}
\end{aligned}
$$

If Equation (45) is defensible as to entropy growth, and gravitons play a role, then the initial temperature may be affected by modeling Gravitons as a particle in a viscous fluid, see Weinberg [2] page 592 with Planck time as of the order of magnitude of a mean free time of collisions of Gravitons in a formation plasma as an imperfect fluid

$$
T^{\beta} \sim\left[\frac{15}{4} \cdot \frac{1}{a} \cdot \frac{\eta}{t_{\text {Plank }}}\right]^{\beta / 4} \propto\left[\frac{15}{4} \cdot \frac{1}{a} \cdot \frac{\eta}{\tau_{\text {graviton }}}\right]^{\beta / 4}
$$

Using this Equation (46), if the viscosity drops, the temperature drops as well. We posit that this will then have immediate consequences for the problem of a multiverse versus a single universe.

\section{Penrose Comology Supposition and Its Tie in to Temperature and Viscosity as Used in Equation (46)}

From Penrose, [3] page 130, we have

$E=8 \pi \cdot T+\Lambda \cdot g$

$E=$ source for gravitational field

$T=$ mass energy density 


$$
g \underset{c c c}{\longrightarrow} \hat{g}=\Omega^{2} g
$$

The single universe CCC has, then, when black holes (up to a million of them) take in space time from an expanding universe, a "reverse" conformal re set which Penrose [3] sets as

$$
\Omega \underset{C C C}{\longrightarrow} \Omega^{-1}
$$

This re set, would tend toward a huge viscosity, as for Equation (46) with a very large initial temperature.

The question is, what would happen to this equation, and temperature if there is a multiverse i.e. the viscosity would drop, and that would lead to low initial pre big bang temperatures, i.e. see the following

$$
\Pi=\frac{d \Omega}{\Omega^{2}-1} \underset{\Omega \underset{C C C}{\longrightarrow} \Omega^{-1}}{\longrightarrow} \operatorname{SAME} \frac{d \Omega}{\Omega^{2}-1}
$$

This will be generalized in the case of a multi verse as follow. Equation (47) is not really altered, but Equation (49) will have a multi verse interpretation, i.e. for the four dimensional "domain" universes we will call $\Omega_{\tilde{j}}$

$$
\Omega_{\text {input-new-cycle }} \simeq \frac{1}{n[\text { number-of-'universes'] }} \cdot \sum_{\tilde{j}=1}^{n[\text { number-of-'universes'] }} \Omega_{\tilde{j}}
$$

Then, there will be a new mapping, of

$$
\Omega_{\tilde{j}}\left[\text { 'our-universe'] } \rightarrow\left(\Omega_{\text {input-new-cycle }}\right)^{-1}\right.
$$

This would, then go to a modification of the Penrose mapping in Equation (50) to read as

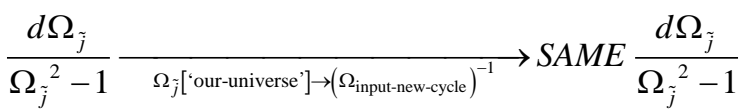

Equation (53) with Equation (52) are in reality a motivation of Ergodic mixing, i.e. a way to have continual re sets of the initial data, for the purpose of having the same initial conditions again and again, i.e. this is a way of a re set of Planck's constant, and of the fine structure constant without the annoyance of the anthropic principle.

The governing principle behind it would be the Ergodic theorem, i.e. see Dye [32], as an averaging process again and again. In doing so, Equation (52) and Equation (53) would through mixing lead to the same re set again and again, but with one major different consequence than in the single repeating universe case, i.e. there would be a low temperature pre big bang, using Equation (43), instead of Equation (42). This would be a way of having a very different answer as to the character of early universe GW generation, with the multiverse having very low GW frequency, even before red shifting of GW, instead of the high frequency GW predicted by Equation (42). Next then, we will examine the consequences of the initial starting point of the multiverse recycled starting point. Note the multiverse would likely entail an initially low viscosity, which says low temperature. 


\section{Re Examining the Question of a "Near Singularity" in a Multiverse}

We follow the recent work of Steven Kenneth Kauffmann [40], which sets an upper bound to concentrations of energy, in terms of how he formulated the following equation put in below as Equation (54). Equation (54) specifies an inter-relationship between an initial radius $R$ for an expanding universe, and a "gravitationally based energy" expression we will call $T_{G}(r)$ which lead to a lower bound to the radius of the universe at the start of the Universe's initial expansion, with manipulations. The term $T_{G}(r)$ is defined via Equation (55) afterwards. We start off with Kauffmann's expression [40]

$$
R \cdot\left(\frac{c^{4}}{G}\right) \geq \int_{\left|r^{\prime \prime}\right| \leq R} T_{G}\left(r+r^{\prime \prime}\right) \mathrm{d}^{3} r^{\prime \prime}
$$

Kauffmann calls $\left(\frac{c^{4}}{G}\right)$ a "Planck force" which is relevant due to the fact we will employ Equation (54) at the initial instant of the universe, in the Planckian regime of space-time. Also, we make full use of setting for small $r$, the following:

$$
T_{G}\left(r+r^{\prime \prime}\right) \approx T_{G=0}(r) \cdot \text { const } \sim V(r) \sim m_{\text {Graviton }} \cdot n_{\text {Initial-entropy }} \cdot c^{2}
$$

i.e. what we are doing is to make the expression in the integrand proportional to information leaked by a past universe into our present universe, with $\mathrm{Ng}$ [8] style quantum infinite statistics use of

$$
n_{\text {Initial-entropy }} \sim S_{\text {Graviton-count-entropy }}
$$

Then Equation (54) will lead to

$$
\begin{aligned}
& R \cdot\left(\frac{c^{4}}{G}\right) \geq \int_{\left|r^{\prime \prime}\right| \leq R} T_{G}\left(r+r^{\prime \prime}\right) \mathrm{d}^{3} r^{\prime \prime} \approx \text { const } \cdot m_{\text {Graviton }} \cdot\left[n_{\text {Initial-entropy }} \sim S_{\text {Graviton-count-entropy }}\right] \\
& \Rightarrow R \cdot\left(\frac{c^{4}}{G}\right) \geq \text { const } \cdot m_{\text {Graviton }} \cdot\left[n_{\text {Initial-entropy }} \sim S_{\text {Graviton-count-entropy }}\right] \\
& \Rightarrow R \geq\left(\frac{c^{4}}{G}\right)^{-1} \cdot\left[\text { const } \cdot m_{\text {Graviton }} \cdot\left[n_{\text {Initial-entropy }} \sim S_{\text {Graviton-count-entropy }}\right]\right]
\end{aligned}
$$

Here, $\left[n_{\text {Initial-entropy }} \sim S_{\text {Graviton-count-entropy }}\right] \sim 10^{5}, m_{\text {Graviton }} \sim 10^{-62}$ grams, and we set Plank length as:

$$
\text { Planck length }=l_{\text {Planck }}=1.616199 \times 10^{-35} \text { meters }
$$

where we set $l_{\text {Planck }}=\sqrt{\frac{\hbar G}{c^{3}}}$ with $R \sim l_{\text {Planck }} \cdot 10^{\alpha}$, and $\alpha>0$. Typically $R \sim l_{\text {Planck }} \times 10^{\alpha}$ is about $10^{3} \cdot l_{\text {Planck }}$ at the outset, when the universe is the most compact. The value of const is chosen based on common assumptions about contributions from all sources of early universe entropy, and will be more rigorously defined in a later paper.

We argue that the above methodology, giving a non zero initial starting point is made especially tendable if one is using a low temperature start, allowing for 
the existence of prior recycling universes gravitons to play a role, i.e. that in the single universe repeated again and again, there would be real issues as to the survival of the graviton allowing for the conclusion as to Equation (57).

\section{Fork the Road. Nonzero Radii of Start of Inflation May Be Linkable to Low Temperature Pre Universe Due to Multiverse. How to Confirm It?}

The author's supposition and argument as to the results of Equation (57) which would be key to identifying if a zero point starting point to inflation was mandatory are akin to the question that gravity and gravitons could exist prior to inflation. If there is a multiverse, i.e. repeatedly, as mentioned in section 12, then the answer is likely yes. Hence, Equation (57) would indicate that a "perfect" singularity is not mandatory. The problem though is that then likely initial relic GW would then be enormously long, i.e. as given in Equation (43) above. This is independent of red shifting.

If one has ultra high gravity waves as created at the big bang, then it is likely that a singularity is mandatory. We also then have referred to Stoica's work as to how this could be squeezed down below the Planck length limit, but it would not be pathological for the reasons stated above. i.e. confirmation of the two alternatives likely hinges upon determination if ultra high GW is indeed part of the pre inflation to inflationary universe's signal heritage.

Figure 1 is pertinent to a single, repeating universe. The author knows of no viable counterpart to Figure 1 in the case of a multiverse. Hence, as for higher dimensions, the author, in lieu of his generalization of the Penrose cosmology conjecture [3] has referenced Appendix $\mathrm{V}$ below which is really akin to the material given in Wesson [34] as to Appendix VIII below, as to a different geometry than Braneworlds as to the multiverse hypothesis. Suitable inquiry should be in terms of if Braneworlds as of Figure 1 can suitably be modified for the multiverse. The author doubts this is possible i.e. if low GW is confirmed as to relic conditions for our particular universe, then if we choose the multiverse, disternment, if Figure 1 has a real generalization to the multiverse, hypothesis is mandatory. The author believes that this will be futile.

Note also, if a single repeating universe is confirmed via relic HFGW, then Figure 1 is probably legitimate and brane world "vibrations" may be necessary for HFGW. If so, then one can as an intellectual inquiry inquire if the generalization to Appendix VIII is then really necessary.

\section{Acknowledgements}

The author particularly appreciates the Mandelbrot example given to him by J. Dickau as a legitimate research inquiry question which he will pursue in future work. The author also thanks his late father, and Dr. Xi Yang of Brookhaven National Laboratory as to imbuing him with the necessary curiosity and physics questions which much later on lead to the singularity issues in this document being investigated. Part of this presentation was also in lectures to Graduate 
Physics Students in Chongqing University, in November 2013.

\section{References}

[1] Park, D.H., Kim, H. and Tamarayan, S. (2002) Nonvanishing Cosmological Constant of Flat Universe in Brane world Scenarios. Physics Letters B, 535, 5-10. https://doi.org/10.1016/S0370-2693(02)01729-X

[2] Weinberg, S. (1972) Gravitation and Cosmology, Principles and Applications of the General Theory of Relativity. Wiley, San Francisco.

[3] Penrose, R. (2010) Cycles of Time. The Bodley Head, London.

[4] Stoica, C. (2012) Beyond the FRWL Big Bang Singularity. http://arxiv.org/pdf/1203.1819.pdf

[5] Beckwith, A.W. (2012) Is Quantum Mechanics Involved at the Start of Cosmological Evolution? Does a Machian Relationship between Gravitons and Gravitinos Answer This Question? Astrophysics, 36, 1019-1021. http://vixra.org/abs/1206.0023

[6] Lee, J.W. (2012) On the Origin of Entropic Gravity and Inertia. Foundations of Physics, 42, 1153-1164. https://doi.org/10.1007/s10701-012-9660-x

[7] Muller, R. and Lousto, A.C. (1995) Entanglement Entropy in Curved Space-Times with Event Horizons. Physical Review D Particles \& Fields, 52, 4512-4517. https://doi.org/10.1103/PhysRevD.52.4512

[8] Ng, Y.J. (2008) Spacetime Foam: From Entropy and Holography to Infinite Statistics and Nonlocality. Entropy, 10, 441-461. https://doi.org/10.3390/e10040441

[9] Durrer, R., et al. (2007) Dynamical Casmir Effect for Gravitons in Bouncing Braneworlds. Physical Review D Particles \& Fields, 76, 653. http://theory.physics.unige.ch/ durrer/papers/casimir.pdf;

[10] Volovik, G.E. (2006) Vacuum Energy: Myths and Realities. International Journal of Modern Physics D, 15, 1987-2010. https://doi.org/10.1142/S0218271806009431

[11] Mitra, A. (2011) Why the Big Bang Model Cannot Describe the Observed Universe Having Pressure and Radiation. Journal of Modern Physics, 2, 1436-1442. https://doi.org/10.4236/jmp.2011.212177

[12] Beckwith, A. (2010) How to Use the Cosmological Schwinger Principle for Energy Flux, Entropy, and "Atoms of Space-Time" to Create a Thermodynamic SpaceTime and Multiverse. Proceedings of 5 th International Workshop DICE2010: Space-Time-Matter-Current Issues in Quantum Mechanics and Beyond, Castiglioncello, 13-17 September 2010, 1-11.

http://iopscience.iop.org/1742-6596/306/1/012064

http://iopscience.iop.org/1742-6596/306/1;jsessionid=A05372A78C18D970BF35F40 A9A863B51.c2

[13] Gryzinski, M. (1959) Classical Theory of Electronic and Ionic Inelastic Collisions. Physical Review, 115, 374-383. https://doi.org/10.1103/PhysRev.115.374

[14] Bruchholz, U. (2009) Derivation of Planck's Constant from Maxwell's Electrodynamics. Progress in Physics, 4, 67.

[15] Bruzchholz, U. (2009) Key Notes on a Geometric Theory of Fields. Progress in Physics, 2, 107-113.

[16] Beckwith, A. (2011) Octonionic Gravity Formation, Its Connections to Micro Physics. Open Journal of Microphysics, 1, 13-18. https://doi.org/10.4236/ojm.2011.11002

[17] Pringle, J. and King, A. (2007) Astrophysical Flows. Cambridge University Press, New York. https://doi.org/10.1017/CBO9780511802201

[18] Padmanabhan, T. (2011) Lessons from Classical Gravity about the Quantum Struc- 
ture of Space-Time. Journal of Physics: Conference Series, 306, Article ID: 012001. http://iopscience.iop.org/1742-6596/306/1/012001 https://doi.org/10.1088/1742-6596/306/1/012001

[19] Gao, C. (2010) A Model of Nonsingular Universe. Entropy2012, 14, 1296-1305.

[20] Dickau, J. (2012) Private Communication to the Author as to Minimum SpaceTime "Length" Provided as of August 7, 2012.

[21] Beckwith, A. (2011) Identifying a Kaluza Klein Treatment of a Graviton Permitting a Deceleration Parameter Q $(Z)$ as an Alternative to Standard DE. Journal of Cosmology, 13, 1-15. http://journalofcosmology.com/BeckwithGraviton.pdf

[22] Surya, S. (2010) In Search of a Covariant Quantum Measure. Journal of Physics. Conference Series, 306, 1-7. http://iopscience.iop.org/1742-6596/306/1/012018

[23] Beckwith, A. (2017) Extra Dimensions, Brane Worlds, and the Vanishing of Axion Contributions to Inflation. Journal of High Energy Physics, Gravitation and Cosmology, 3, 285-295. http://journalofcosmology.com/BeckwithGraviton.pdf https://doi.org/10.4236/jhepgc.2017.32024

[24] Koury, J., Steinhardt, P.J. and Turkok, N. (2004) Designing Cyclic Universe Models. Physics Review Letters, 92, Article No. 031302.

[25] Koury, J., Steinhardt, P.J. and Turkok, N. (2003) Inflation versus Cyclic Predictions for Spectral Tilt. Physics Review Letters, 91, Article No. 161301.

[26] Lloyd, S. (2002) Computational Capacity of the Universe. Physics Review Letters, 88, Article ID: 237901. https://doi.org/10.1103/physrevlett.88.237901

[27] Beckwith, A.W. (2005) Symmetries in Evolving Space Time from Prior to Present Universes. Mathematics, 1-23.

[28] Barvinsky, A., Kamenschick, A. and Yu, A. (2006) Thermodynamics from Nothing: Limiting the Cosmological Constant Landscape. Physical Review D Particles \& Fields, 74, 421-432. https://doi.org/10.1103/PhysRevD.74.121502

[29] Padmanabhan, T. (2006) An Invitation to Astro Physics. World Scientific Publishing, Singapore City. https://doi.org/10.1142/6010

[30] Padmanabhan, T. (2005) Understanding Our Universe: Current Status and Open Issues. In: Ashtekar, A., Ed., 100 Years of Relativity Space-Time Structure: Einstein and Beyond, World Scientific Publishing, Singapore City.

[31] Crowell, L. (2005) Quantum Fluctuations of Space-Time. World Scientific Series in Contemporary Chemical Physics, 25, 388.

[32] Dye, H. (1964) On the Ergodic Mixing Theorem. Transactions of the American Mathematical Society, 118, 123-130.

http://www.ams.org/journals/tran/1965-118-00/S0002-9947-1965-0174705-8/S00029947-1965-0174705-8.pdf https://doi.org/10.1090/S0002-9947-1965-0174705-8

[33] Penrose, R. (2011) Cycles of Time: An Extraordinary New View of the Universe. Knopf Doubleday Publishing Group, New York.

[34] Wesson, P. (1999) Space-Time-Matter, Modern Kaluza Klein Theory. World Scientific Publishing, Singapore City. https://doi.org/10.1142/3889

[35] Peacock, J. (2002) Cosmological Physics. Cambridge University Press, Cambridge.

[36] Grischuck, L. (2006) Personal Communication to the Author. John Wheeler School of Astrophysics, Erice.

[37] Giovannini, M. (2008) A Primer on the Physics of the Cosmic Microwave Background. World Scientific Publishing, Singapore City. https://doi.org/10.1142/6730

[38] Misner, C., Thorne, K. and Wheeler, J. (1973) Gravitation. W.H. Freeman, and Company, San Francisco. 
[39] Samtleben, D., Staggs, S. and Winstein, S.B. (2007) The Cosmic Microwave Background for Pedestrians: A Review for Particle and Nuclear Physicists. Annual Review of Nuclear \& Particle Science, 57, 245-283.

https://doi.org/10.1146/annurev.nucl.54.070103.181232

[40] Kauffman, S. A Self Gravitational Upper Bound on Localized Energy Including that of Virtual Particles and Quantum Fields, Which Yield a Passable Dark Energy Density Estimate. Physics, 5. http://arxiv.org/abs/1212.0426 


\section{Appendix I. Fjortoft Theorem}

A necessary condition for instability is that if $Z_{*}$ is a point in spacetime for which $\frac{\mathrm{d}^{2} U}{\mathrm{~d} z^{2}}=0$ for any given potential $U$, then there must be some value $z_{0}$ in the range $z_{1}<z_{0}<z_{2}$ such that

$$
\left.\frac{\mathrm{d}^{2} U}{\mathrm{~d} z^{2}}\right|_{z_{0}} \cdot\left[U\left(z_{0}\right)-U\left(z_{*}\right)\right]<0
$$

For the proof, see [17] and also consider that the main discussion is to find instability in a physical system which will be described by a given potential $U$. Next, we will construct in the boundary of the EW era, a way to come up with an optimal description for $U$.

\section{Appendix II. Constructing an Appropriate Potential for Using Fjortoft Theorem in Cosmology for the Early Universe Cannot Be Done. We Show Why}

To do this, we will look at Padamanabhan [18] and his construction of (in Dice 2010) of thermodynamic potentials he used to have another construction of the Einstein GR equations. To start, Padamanabhan [18] wrote:

If $P_{c d}^{a b}$ is a so called Lovelock entropy tensor, and $T_{a b}$ a stress energy tensor

$$
\begin{aligned}
& U\left(\eta^{a}\right)=-4 \cdot P_{a b}^{c d} \nabla_{c} \eta^{a} \nabla_{d} \eta^{b}+T_{a b} \eta^{a} \eta^{b}+\lambda(x) g_{a b} \eta^{a} \eta^{b} \\
& =U_{\text {gravity }}\left(\eta^{a}\right)+U_{\text {matter }}\left(\eta^{a}\right)+\lambda(x) g_{a b} \eta^{a} \eta^{b} \\
& \Leftrightarrow U_{\text {matter }}\left(\eta^{a}\right)=T_{a b} \eta^{a} \eta^{b} ; U_{\text {gravity }}\left(\eta^{a}\right)=-4 \cdot P_{a b}^{c d} \nabla_{c} \eta^{a} \nabla_{d} \eta^{b}
\end{aligned}
$$

We now will look at

$$
\begin{aligned}
& U_{\text {matter }}\left(\eta^{a}\right)=T_{a b} \eta^{a} \eta^{b} ; \\
& U_{\text {gravity }}\left(\eta^{a}\right)=-4 \cdot P_{a b}^{c d} \nabla_{c} \eta^{a} \nabla_{d} \eta^{b}
\end{aligned}
$$

So happens that in terms of looking at the partial derivative of the top (1) equation, we are looking at

$$
\frac{\partial^{2} U}{\partial\left(\eta^{a}\right)^{2}}=T_{a a}+\lambda(x) g_{a a}
$$

Thus, we then will be looking at if there is a specified $\eta_{*}^{a}$ for which the following holds.

$$
\begin{aligned}
{\left[\frac{\partial^{2} U}{\partial\left(\eta^{a}\right)^{2}}=\right.} & \left.T_{a a}+\lambda(x) g_{a a}\right]_{\eta_{0}^{a}} *\left[-4 \cdot P_{a b}^{c d}\left(\nabla_{c} \eta_{0}^{a} \nabla_{d} \eta_{0}^{b}-\nabla_{c} \eta_{*}^{a} \nabla_{d} \eta_{*}^{b}\right)\right. \\
& \left.+T_{a b} \cdot\left[\eta_{0}^{a} \eta_{0}^{b}-\eta_{*}^{a} \eta_{*}^{b}\right]+\lambda(x) g_{a b} \cdot\left[\eta_{0}^{a} \eta_{0}^{b}-\eta_{*}^{a} \eta_{*}^{b}\right]\right]<0
\end{aligned}
$$

What this is saying is that there is no unique point, using this $\eta_{*}^{a}$ for which (4) holds. Therefore, we say there is no official point of instability of $\eta_{*}^{a}$ due to

(3). The Lagrangian structure of what can be built up by the potentials given in (3) with respect to $\eta_{*}^{a}$ mean that we cannot expect an inflection point with re- 
spect to a $2^{\text {nd }}$ derivative of a potential system. Such an inflection point designating a speed up of acceleration due to DE exists a billion years ago [19] [23]. Also note that the reason for the failure for (4) to be congruent to Fjoroft's theorem is due to

$$
\left[\frac{\partial^{2} U}{\partial\left(\eta^{a}\right)^{2}}=T_{a a}+\lambda(x) g_{a a}\right] \neq 0 \text {, for } \forall \eta_{*}^{a} \text { choices }
$$

\section{Appendix III. Details as to Forming Crowell's Time Dependent Wheeler De Witt Equation, and Its Links to Worm Holes}

This will be to show some things about the worm hole we assert the instanton traverses en route to our present universe. From Crowell [31]

$$
-\frac{1}{\eta r} \frac{\partial^{2} \Psi}{\partial r^{2}}+\frac{1}{\eta r^{2}} \cdot \frac{\partial \Psi}{\partial r}+r R^{(3)} \Psi=(r \eta \phi-r \ddot{\phi}) \cdot \Psi
$$

This has when we do it $\phi \approx \cos (\omega \cdot t)$, and frequently $R^{(3)} \approx$ constant, so then we can consider

$$
\phi \cong \int_{0}^{\infty} \mathrm{d} \omega\left[a(\omega) \cdot \mathrm{e}^{i k_{\sigma} x^{\mu}}-a^{+}(\omega) \cdot \mathrm{e}^{-i k_{\varpi} x^{\mu}}\right]
$$

In order to do this, we can write out the following with regards to the solutions to Equation (1) put up above.

$$
\begin{aligned}
C_{1}= & \eta^{2} \cdot\left(4 \cdot \sqrt{\pi} \cdot \frac{t}{2 \omega^{5}} \cdot J_{1}(\omega \cdot r)+\frac{4}{\omega^{5}} \cdot \sin (\omega \cdot r)+(\omega \cdot r) \cdot \cos (\omega \cdot r)\right) \\
& +\frac{15}{\omega^{5}} \cos (\omega \cdot r)-\frac{6}{\omega^{5}} \operatorname{Si}(\omega \cdot r)
\end{aligned}
$$

And

$$
C_{2}=\frac{3}{2 \cdot \omega^{4}} \cdot(1-\cos (\omega \cdot r))-4 \mathrm{e}^{-\omega \cdot r}+\frac{6}{\omega^{4}} \cdot \operatorname{Ci}(\omega \cdot r)
$$

This is where $\operatorname{Si}(\omega \cdot r)$ and $\operatorname{Ci}(\omega \cdot r)$ refer to integrals of the form $\int_{-\infty}^{x} \frac{\sin \left(x^{\prime}\right)}{x^{\prime}} d x^{\prime}$ and $\int_{-\infty}^{x} \frac{\cos \left(x^{\prime}\right)}{x^{\prime}} d x^{\prime}$. It so happens that this is for forming the wave functional permitting an instanton forming, while we next should consider if or not the instanton so farmed is stable under evolution of space time leading up to inflation. We argue here that we are forming an instanton whose thermal energy is focused into a wave functional which is in the throat of the worm hole up to a thermal discontinuity barrier at the onset, and beginning of the inflationary era.

\section{Appendix IV. The D'Albembertain Operation in an Equation of Motion for Emergent Scalar Fields}

We begin with the D'Albertain operator as part of an equation of motion for an emergent scalar field. We refer to the Penrose potential (with an initial assump- 
tion of Euclidian flat space for computational simplicity) to account for, in a high temperature regime an emergent non zero value for the scalar field $\phi$ due to a zero effective mass, at high temperatures [32].

When the mass approaches far lower values, it, a non zero scalar field re appears.

Leading to $\phi \underset{T \rightarrow 2.7 \text { Kelvin }}{\longrightarrow} \varepsilon^{+} \approx 0^{+}$as a vanishingly small contribution to cosmological evolution.

Let us now begin to initiate how to model the Penrose quintessence scalar field evolution equation. To begin, look at the flat space version of the evolution equation

$$
\ddot{\phi}-\nabla^{2} \phi+\frac{\partial V}{\partial \phi}=0
$$

This is, in the Friedman-Walker metric using the following as a potential system to work with, namely:

$$
\begin{aligned}
V(\phi) & \sim-\left[\frac{1}{2} \cdot\left(M(T)+\frac{\mathfrak{R}}{6}\right) \phi^{2}+\frac{\tilde{a}}{4} \phi^{4}\right] \\
& \equiv-\left[\frac{1}{2} \cdot\left(M(T)+\frac{\kappa}{6 a^{2}(t)}\right) \phi^{2}+\frac{\tilde{a}}{4} \phi^{4}\right]
\end{aligned}
$$

This is pre supposing $\kappa \equiv \pm 1,0$, that one is picking a curvature signature which is compatible with an open universe.

That means $\kappa=-1,0$ as possibilities. So we will look at the $\kappa=-1,0$ values. We begin with.

$$
\begin{aligned}
& \ddot{\phi}-\nabla^{2} \phi+\frac{\partial V}{\partial \phi}=0 \\
& \Rightarrow \phi^{2}=\frac{1}{\tilde{a}} \cdot\left\{c_{1}^{2}-\left[\alpha^{2}+\frac{\kappa}{6 a^{2}(t)}+M(T)\right]\right\} \\
& \Leftrightarrow \phi \equiv \mathrm{e}^{-\alpha \cdot r} \exp \left(c_{1} t\right)
\end{aligned}
$$

We find the following as far as basic phenomenology, namely

$$
\begin{aligned}
& \phi^{2}=\frac{1}{\tilde{a}} \cdot\left\{c_{1}^{2}-\left[\alpha^{2}+\frac{\kappa}{6 a^{2}(t)}+\left(M(T) \approx \varepsilon^{+}\right)\right]\right\} \underset{M(T \sim \text { high }) \rightarrow 0}{\longrightarrow} \phi^{2} \neq 0 \\
& \phi^{2}=\frac{1}{\tilde{a}} \cdot\left\{c_{1}^{2}-\left[\alpha^{2}+\frac{\kappa}{6 a^{2}(t)}+\left(M(T) \neq \varepsilon^{+}\right)\right]\right\} \underset{M(T \sim \text { Low }) \neq 0}{\longrightarrow} \phi^{2} \approx 0
\end{aligned}
$$

The difference is due to the behavior of $M(T)$. We use $M(T)$ axion mass $m_{a}(T)$ in asymptotic limits with

$$
m_{a}(T) \cong 0.1 \cdot m_{a}(T=0) \cdot\left(\Lambda_{Q C D} / T\right)^{3.7}
$$

\section{Appendix V. Interesting Speculation. Does There Exist a Five Dimensional Version of an Instanton in the Worm Hole Transition Regime?}

We will attempt to build the contribution as to a Reissner-Nordstrom metric 
embedded in a five dimensional space-time metric, and see if this satisfied. i.e. look at (1) below This allows us to determine, using of the Risessner-Nordstrom metric as given, by Kip Thorne, Wheeler, and Misner [37], for an added cosmological "constant" $\Lambda$ and "charge" $Q$. This will be shown to lead to [33]

$$
\begin{aligned}
M_{g}(r)= & \int\left[T_{0}^{0}-\left(T_{1}^{2}+2 \cdot T_{2}^{2}\right)\right] \cdot \sqrt{-g_{4}} \mathrm{~d} V_{3} \\
\approx & \pi \cdot c_{1}^{2} \cdot\left[\frac{r^{3}}{3}-2 M \cdot \frac{r^{2}}{2}+Q \cdot r-\frac{\Lambda}{15} \cdot r^{5}\right] \\
& +4 \pi \cdot c_{1} \cdot\left[r^{2}-8 \cdot M \cdot r-\frac{\Lambda}{3} \cdot r^{4}\right] \underset{r \rightarrow \delta}{\longrightarrow} \varepsilon^{+} \approx 0
\end{aligned}
$$

To do this, we start off with the following space time line metric in five dimensions. This is a modification of Wesson's book [33]

$$
\mathrm{d} S_{5-\operatorname{dim}}=[\exp (i \pi / 2)] \cdot\left\{\mathrm{e}^{2 \Phi(r)} \mathrm{d} t^{2}+\mathrm{e}^{2 \tilde{\Lambda}(r)} \mathrm{d} r^{2}+R^{2} \mathrm{~d} \Omega^{2}\right\}+(-1) \cdot \mathrm{e}^{\mu} \mathrm{d} l^{2}
$$

We claim that what is in the \{\} brackets is just the Reissner-Nordstrom line metric in four dimensional space. The parameters in the \{\} bracket are linked to the Reissner-Nordstrom metric via

$$
\mathrm{e}^{2 \Phi(r)}=\left(1-\frac{2 M}{r}+\frac{Q^{2}}{r^{2}}\right)
$$

And

$$
\mathrm{e}^{2 \tilde{\Lambda}(r)}=\left(1-\frac{2 M}{r}+\frac{Q^{2}}{r^{2}}\right)^{-1}
$$

And this is assuming that $R \sim r$ as well as using $\mu \approx c_{1} \cdot r$ with a maximum value topped off by a Planck's length value due to

$\mu_{\text {Maximum }} \approx c_{1} \cdot r_{\text {Maximum }} \sim l_{P} \equiv 10^{-35} \mathrm{~cm}$. So being the case, we get the following stress tensor values:

$$
\begin{array}{r}
T_{0}^{0}=\left(\frac{-1}{8 \pi}\right) \cdot\left(1-\frac{2 M}{r}+\frac{Q^{2}}{r^{2}}-\frac{\Lambda}{3} r^{2}\right) \cdot\left(\frac{c_{1}^{2}}{4}+\frac{c_{1}}{r}+\frac{c_{1}}{4} \cdot\left[\frac{\frac{2 M}{r^{2}}-\frac{2 Q}{r^{3}}-\frac{2 \Lambda r^{2}}{3}}{1-\frac{2 M}{r}+\frac{Q^{2}}{r^{2}}-\frac{\Lambda}{3} r^{2}}\right]\right) \\
T_{1}^{1}=\left(\frac{-1}{8 \pi}\right) \cdot\left(1-\frac{2 M}{r}+\frac{Q^{2}}{r^{2}}-\frac{\Lambda}{3} r^{2}\right) \cdot\left(\frac{c_{1}}{r}+\frac{c_{1}}{4} \cdot\left[\frac{\frac{2 M}{r^{2}}-\frac{2 Q}{r^{3}}-\frac{2 \Lambda r^{2}}{3}}{1-\frac{2 M}{r}+\frac{Q^{2}}{r^{2}}-\frac{\Lambda}{3} r^{2}}\right]\right) \\
T_{2}^{2}=T_{3}^{3}=\left(\frac{-1}{8 \pi}\right) \cdot\left(1-\frac{2 M}{r}+\frac{Q^{2}}{r^{2}}-\frac{\Lambda}{3} r^{2}\right) \cdot\left(\frac{c_{1}^{2}}{4}+\frac{c_{1}}{r}+\frac{c_{1}}{2} \cdot\left[\frac{\frac{2 M}{r^{2}}-\frac{2 Q}{r^{3}}-\frac{2 \Lambda r^{2}}{3}}{1-\frac{2 M}{r}+\frac{Q^{2}}{r^{2}}-\frac{\Lambda}{3} r^{2}}\right]\right)
\end{array}
$$

Furthermore, we get the following determinant value

$$
\sqrt{-g_{4}}=\left(1-\frac{2 M}{r}+\frac{Q^{2}}{r^{2}}-\frac{\Lambda}{3} r^{2}\right)
$$


All these together lead to Equation (1) being satisfied. Let us now see how this same geometry contributes to a worm hole bridge and a solution as to forming the instanton flux wave functional between a prior to a present universe. The Reissner-Nordstrom metric permits us to have a radiation dominated "matter" solution whose matter "contribution" drops off rapidly as the spatial component of geometry goes to zero. This is in tandem with radiation pressure and density falling off rapidly, as we leave the center of such a purported soliton/instanton. This is extremely useful because it ties in with the notion of fractional branes contributing to entropy calculations. In fact it is useful to state that these two notions dove tail with each other quite closely. The only difference is that the construction above does not in itself lend to the complexity of what we would observe, which is in itself a multiple-joined net work of charge centers and of shifting geometry.

\section{Appendix VI. Basic Physics of Achieving Minimum Precision in CMBR Power Spectra Measurements}

Begin first of all looking at

$$
\frac{\Delta T}{T} \equiv \sum_{l, m} a_{l m} Y_{l, m}(\theta, \phi)
$$

This leads to consider what to do with

$$
C_{l}=\left\langle\left|a_{l, m}\right|^{2}\right\rangle
$$

Samtleben et al. [38] consider then what the experimental variance in this power spectrum, to the tune of an achievable precision given by

$$
\frac{\Delta C_{l}}{C_{l}}=\sqrt{\frac{2}{2 l+1}} \cdot\left(\frac{1}{\sqrt{f_{\text {sky }}}}+\frac{4 \pi \cdot\left(\Delta T_{\text {exp }}\right)^{2}}{C_{l}} \cdot \sqrt{f_{\text {sky }}} \cdot \mathrm{e}^{l^{2} \sigma_{b}^{2}}\right)
$$

$f_{\text {sky }}$ is the fraction of the sky covered in the measurement, and $\Delta T_{\exp }$ is a measurement of the total experimental sensitivity of the apparatus used. Also $\sigma_{b}$ is the width of a beam, while we have a minimum value of $l_{\min } \approx(1 / \Delta \Theta)$ which is one over the fluctuation of the angular extent of the experimental survey i.e. contributions to $C_{l}$ uncertainty from sample variance is equal to contributions to $C_{l}$ uncertainty from noise. The end result is

$$
4 \pi \cdot f_{\text {sky }}=C_{l} \cdot\left(\exp \left[-l^{2} \sigma^{2}\right]\right) /(\Delta T)^{2}
$$

\section{Appendix VII. Vacuum Fluctuations Which May Occur: Cosmological Perturbation Theory and Tensor Fluctuations (Gravity Waves)}

Durrer [9] reviews how to interpret $C_{l}$ in the region where we have $2<l<100$, roughly in the region of the Sachs-Wolf contributions due to gravity waves. We begin first of all by looking at an initial perturbation, using a scalar field treat- 
ment of the "Bardeen potential" $\Psi$ This can lead us to put up, if $H_{i}$ is the initial value of the Hubble expansion parameter

$$
k^{3}|\Psi|^{2} \cong\left(\frac{H_{i}}{M_{P}}\right)^{2}
$$

And

$$
\left\langle|\Psi|^{2}\right\rangle \cdot k^{3}=A^{2} k^{n-1} \cdot \eta_{0}^{n-1}
$$

Here we are interpreting $A=$ amplitude of metric perturbations at horizon scale, and we set $k=1 / \eta_{0}$, where $\eta$ is the conformal time, according to $\mathrm{d} t \equiv a \mathrm{~d} \eta=$ physical time, where we have $a$ as the scale factor. Then for $2<l<100$, and $-3<n<3$, and a pure power law given by

$$
\left\langle|H(k, \eta=1 / k)|^{2}\right\rangle \cdot k^{3}=A_{T}^{2} k^{n_{T}} \cdot \eta_{0}^{-n_{T}}
$$

We get for tensor fluctuation, i.e. gravity waves,, and a scale invariant spectrum with $n_{T}=0$

$$
C_{l}^{(T)} \approx \frac{A_{T}^{2}}{(l+3) \cdot(l-2)} \cdot \frac{1}{15 \pi}
$$

\section{Apppendix VIII. Toward a 5 Dimensional Geometry Which May Be Pertinent to the Five Dimensional Multiverse Hypothesis: From Wesson [33]}

$$
\mathrm{d} S_{5-\text { Dim }}^{2}=l^{2}\left[\mathrm{~d} t^{2}-\sum_{j=1}^{3}\left(\exp \left[i\left(\omega \cdot t+k_{j} x_{j}\right)\right]\right) \cdot \mathrm{d} x_{j}\right]-L^{2} \mathrm{~d} l^{2}
$$

Here in this setting, $L$ is the length of the dimension in $5 \mathrm{dim}$. The other "lengths" are from 4D contained dimension in the line element in 5D. $j=1,2,3$ corresponds to $x, y, z$, while $t$ is for the time. Furthermore, we also have that the five dimensional cosmological "constant" is negative

$$
\Lambda_{5-\mathrm{D}}=-\frac{3 \cdot \omega^{2}}{4 l^{2}}
$$

Whereas we also have

$$
\begin{gathered}
8 \pi T_{00}=-\frac{3 \cdot \omega^{2}}{4} \\
8 \pi T_{j j}=\frac{3 \omega^{2}}{4} \cdot \exp \left[i \cdot\left(\omega \cdot t+k_{j} x_{j}\right)\right] \\
R=12 / l^{2} L^{2}=\text { radius of curvature } \longrightarrow 0 \\
8 \pi p=-8 \pi \rho=\frac{3 \cdot \omega^{2}}{4 l^{2}}
\end{gathered}
$$


Submit or recommend next manuscript to SCIRP and we will provide best service for you:

Accepting pre-submission inquiries through Email, Facebook, LinkedIn, Twitter, etc. A wide selection of journals (inclusive of 9 subjects, more than 200 journals)

Providing 24-hour high-quality service

User-friendly online submission system

Fair and swift peer-review system

Efficient typesetting and proofreading procedure

Display of the result of downloads and visits, as well as the number of cited articles Maximum dissemination of your research work

Submit your manuscript at: http://papersubmission.scirp.org/

Or contact jhepgc@scirp.org 